\title{
Canadian Linguistic Association 2016 National Achievement Award Distinguished researcher and leader Dr. Marguerite MacKenzie
}

The 2016 laureate of the National Achievement Award of the Canadian Linguistic Association is Dr. Marguerite MacKenzie of Memorial University of Newfoundland. Dr. MacKenzie's nomination was chosen from a distinguished set of nominations for her significant contributions to the study of Aboriginal languages in Canada, her involvement in the Canadian linguistics community, and her leadership as a teacher, mentor, researcher, and administrator.

Dr. MacKenzie is the undisputed authority on the grammar of the East CreeInnu-Naskapi language continuum in the Algonquian language family. She has built a longstanding relationship with the Innu, Naskapi, and East Cree communities, beginning with the field research she conducted for her 1982 Ph.D. thesis and continuing for over four decades. Her scholarly research on phonological, lexical, and grammatical systems in these languages has resulted in an impressive list of reference works in print and on-line, including two authoritative trilingual dictionaries for InnuEnglish-French (http://www.innu-aimun.ca/) and for East Cree-English-French (http://dictionary.eastcree.org), glossaries of medical and legal terminology, toponymic resources, and several collections of stories.

Dr. MacKenzie has contributed years of leadership to linguistics in Canada. Currently professor emerita at Memorial University of Newfoundland, she served her university as department head, program director, researcher, mentor of colleagues, and student advisor. She served the Canadian Linguistic Association as treasurer for five years, and she continues as a contributor on committees of SSHRC. Dr. MacKenzie was the 2013 recipient of SSHRC's Impact Award in the Insight category.

Dr. MacKenzie presented a plenary talk about her research at the annual meeting of the Canadian Linguistic Association at the Congress of the Humanities and Social Sciences in Calgary.

Contact information: Dr. Marguerite MacKenzie_mmackenz@mun.ca Canadian Linguistic Association National Achievement Award http://cla-acl.ca/?page_id=828 


\section{Association canadienne de linguistique Prix national d'excellence 2016 Chercheure distinguée et leader Mme Marguerite MacKenzie}

La lauréate du Prix national d'excellence décerné par l'Association canadienne de linguistique pour l'année 2016 est Mme Marguerite MacKenzie de l'Université Memorial de Terre-Neuve. Parmi un groupe de nominations des plus distinguées, la candidature de Mme MacKenzie a été retenue en raison de ses importantes contributions à l'étude des langues autochtones du Canada, de son implication dans la communauté canadienne de linguistique et de son leadership en tant qu'enseignante, mentor, chercheure et administratrice.

Mme MacKenzie est une autorité incontestée quant à la grammaire du continuum linguistique cris de l'est-innu-naskapi de la famille des langues algonquiennes. De plus, depuis l'époque de ses travaux de terrain pour l'obtention du doctorat $(\mathrm{PhD}$ en 1982) et tout au long de sa carrière qui couvre plus de quatre décennies, Mme MacKenzie a su développer et maintenir des rapports stables et profitables avec les communautés innues, naskapies et cries. Ses recherches sur les systèmes phonologiques, morphologiques et grammaticaux du continuum cris de l'est-innu-naskapi ont fait l'objet de nombreuses publications (papier et en ligne). On note des dictionnaires trilingues - innu-anglais-français (http://www.innu-aimun.ca/,) et cris de l'estanglais-français (http://dictionary.eastcree.org), des glossaires terminologiques médical et juridique, des répertoires toponymiques et plusieurs anthologies de contes.

Grâce à son leadership, Mme MacKenzie a aussi grandement contribué à l'avancement de la linguistique au Canada. Présentement professeure émérite à l'Université Memorial de Terre-Neuve, elle a été au service de cette institution en tant que chef de département, directrice des études, chercheure, mentor auprès de ses collègues et conseillère auprès des étudiants et étudiantes. Elle a aussi été trésorière de l'Association canadienne de linguistique pendant cinq ans et elle continue de contribuer à sa discipline en tant que membre des comités d'évaluation du CRSH. En 2013, Mme MacKenzie a reçu le prix Impact du CRSH dans la catégorie Savoir.

Mme MacKenzie a présenté une allocution plénière au Congrès annuel de l'Association canadienne de linguistique à Calgary, lors du Congrès des sciences humaines 2016.

Contact : Mme Marguerite MacKenziermmackenz@mun.ca

Prix national d'excellence de l'Association canadienne de linguistique

http://cla-acl.ca/?page_id=828 
CLA president Władysław Cichocki congratulates the recipient of the 2016 National Achievement Award, Marguerite MacKenzie

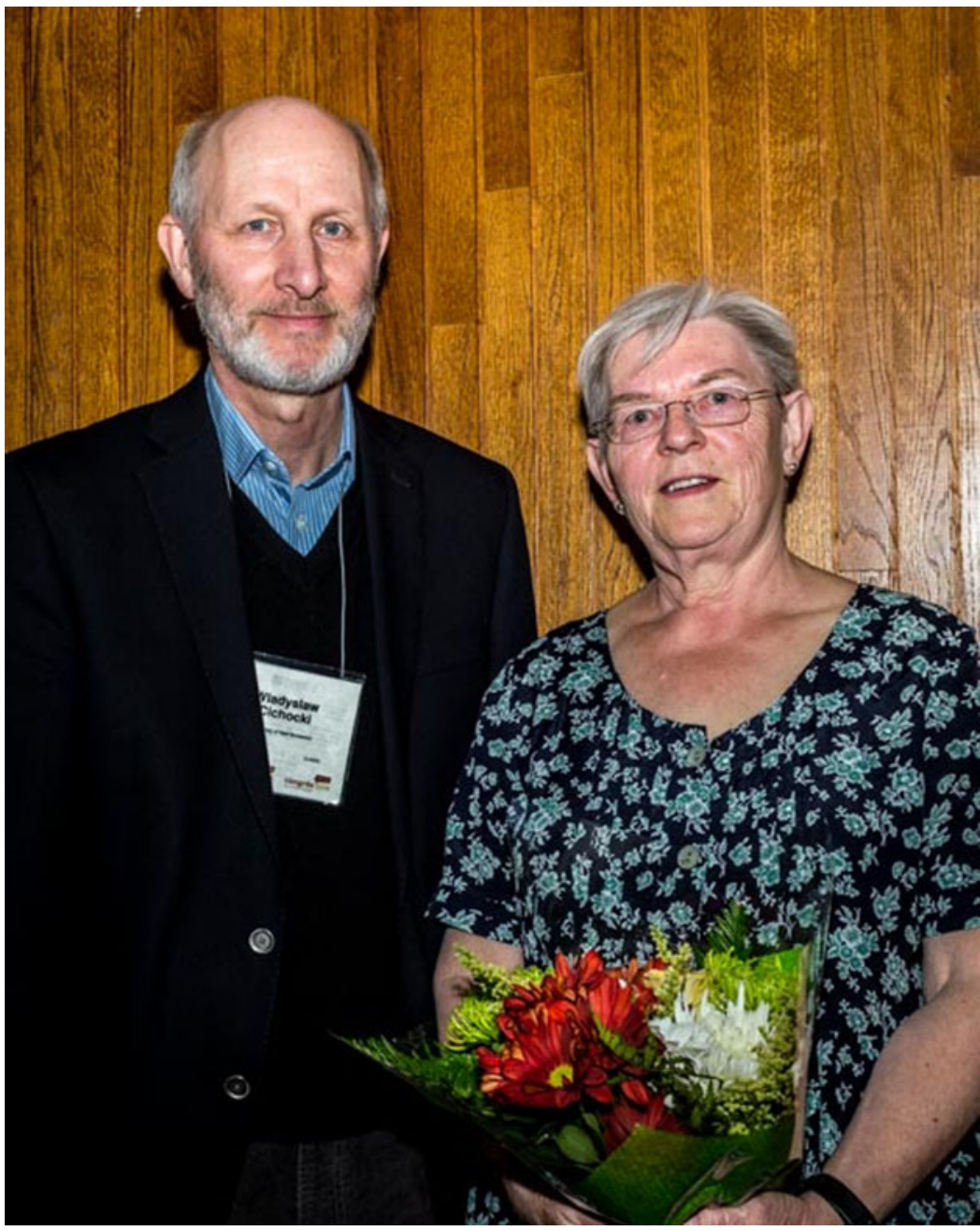

Le président de l'ACL, Władysław Cichocki, félicite la récipiendaire du Prix d'excellence national 2016, Marguerite MacKenzie 